Revista lus et Praxis, Año 25, No 2, 2019, pp. 341 - 358

ISSN 0717 - 2877

Universidad de Talca - Facultad de Ciencias Jurídicas y Sociales

Algunos problemas del régimen de propiedad intelectual de las obtenciones vegetales en Chile

Ricardo Concha M.

Trabajo recibido el 8 de agosto de 2018 y aprobado el 23 de diciembre de 2018

\title{
Algunos problemas del régimen de propiedad intelectual de las obtenciones vegetales en Chile
}

\author{
PROBLEMS OF THE INTELLECTUAL PROPERTY LAW OF PLANT VARIETIES \\ PROTECTION IN CHILE
}

Ricardo Concha M.*

\section{RESUMEN}

En este trabajo se tratan algunos de los problemas que existen en cuanto al derecho del obtentor de variedades vegetales en Chile, específicamente con respecto al objeto y el régimen jurídico de la institución, acusándose el vacío existente en el campo de las acciones protectoras en el ámbito civil y las consecuencias de tal carencia. Se examina si en la dogmática y en la jurisprudencia se ha dado alguna respuesta certera con respecto al problema, o bien si, lejos de ello, existe un panorama marcado por la incerteza jurídica.

\section{ABSTRACT}

This paper intends to highlight some questions that may arise regarding the breeder's right of plant varieties in the field of Chilean civil law and more specifically on the regulation or legal regime of the institution and development on protective actions in the field civil. To thus analyze whether in the dogmatic and jurisprudence have given an answer around the area or far from it have caused a landscape marked by legal uncertainty.

PaLABRAs CLAVE

propiedad intelectual, obtenciones vegetales

KEY WORDS

intellectual property rights, plant varieties protection

\section{Introducción}

Este artículo plantea un análisis de algunas cuestiones de discusión incipiente acerca del derecho del obtentor de variedades vegetales ${ }^{1}$ en el derecho chile-

\footnotetext{
* Profesor del Departamento de Derecho Privado, Facultad de Ciencias Jurídicas y Sociales, Universidad de Concepción, Chile. Correo electrónico: ricardo.concha@udec.cl. Se agradece al plan de incentivo a la publicación de la Facultad de Ciencias Jurídicas y Sociales de la Universidad de Concepción.

1 En cuanto qué son las variedades vegetales como categoría taxonómica (no jurídica), García (2017a), pp. 49-50, indica que "La innovación vegetal busca obtener plantas con unas características determinadas que obedezcan a las necesidades humanas (mayor productividad, resistencia a plagas y enfermedades, mayor belleza de las plantas ornamentales, etcétera). Pues bien, cuando un conjunto de plantas presenta unas características uniformes, que la distinguen de las demás y que son caracteres
} 
no. Este trabajo parte con la exposición sintética de la normativa que regula la materia, en lo que se refiere a los conceptos esenciales, el contenido del derecho, y sus limitaciones. Seguidamente se exponen los fundamentos que se han dado por los instrumentos internacionales y por la doctrina comparada como justificación de la existencia de este derecho. Después se discute el objeto de este derecho, para luego acusar los vacíos normativos que presenta la ley chilena, tratándose estos problemas también en relación con la todavía incipiente jurisprudencia que en este ámbito existe en Chile.

\section{Normativa}

La protección de las variedades vegetales en Chile se halla en la Ley № 19.342, de 1994, que "regula derechos de obtentores de nuevas variedades vegetales" ${ }^{2}$, régimen jurídico especial y sui generis que en términos generales sigue el Convenio UPOV de 1978, regulando los derechos del obtentor de manera similar a otras instituciones de propiedad intelectual o industrial, dado que se puede entender que la protección al obtentor vegetal es del orden de la propiedad intelectual en el sentido lato del término, como se verá en el curso de este trabajo.

El convenio de la Unión Internacional para la Protección de las Obtenciones Vegetales, conocido como UPOV, ratificado por Chile en 1996 en la versión de

estables, estamos ante una variedad vegetal. El concepto de variedad vegetal es, pues, una categoría de clasificación de las plantas. Y como es notorio, la ciencia que se ocupa de la clasificación del reino vegetal es la taxonomía, ciencia que trata, en general, de los principios de la clasificación de los seres vivos [...]. Probablemente, el taxón más conocido (fuera del ámbito científico) sea el de especie. Porque dado que la especie es un taxón que se encuentra en la escala inferior, las plantas de una misma especie se parecen mucho entre sí, con la peculiaridad de que las plantas de distintas especies (como por ejemplo el trigo y el peral), no pueden cruzarse naturalmente. No obstante, dentro de una misma especie las plantas pueden presentar unas características muy distintas entre sí. Piénsese, por ejemplo, en los múltiples tipos de árboles de peral, que presentan características diferentes. Es posible, por lo tanto, seguir clasificando las plantas dentro de una misma especie. Y cada uno de los grupos que se distinguen dentro de una misma especie constituye una variedad vegetal. En cambio, dentro de una misma variedad vegetal ya no es posible hacer más clasificaciones y lo que existe son plantas con unas mismas características".

${ }^{2}$ La ley vigente no es la primera normativa sobre la materia; la primera se estableció mediante el Decreto Ley N $N^{\circ} 1.764$, de 1977, donde se denominaba "propiedad varietal".

La Ley N 19.342 data de 1994 y se encuentra armonizada en términos generales con el Convenio de la Unión para la Protección de Variedades Vegetales, de 1978. Mediante Mensaje 453-356, de 22 de diciembre de 2008, durante la primera presidencia de Michelle Bachelet, se proponía una modificación de esta ley, la que tenía por objeto adecuar la normativa interna de Chile al Convenio de la Unión para la Protección de Variedades Vegetales de 1991, denominado UPOV 91. Dicho proyecto, conocido mediáticamente como "Ley Monsanto", fue retirado del Congreso Nacional durante la segunda presidencia de Michelle Bachelet. Para una perspectiva crítica del convenio UPOV 91, véase BARRERA y Donoso (2013), p. 219. 
1978, establece el régimen jurídico del derecho de las obtenciones vegetales, de orden internacional, al que, en principio y en términos generales, obedece la reglamentación de la materia en Chile, desde dicha ratificación, aunque la ley chilena no se adecua totalmente a dicho convenio ${ }^{3}$.

En Chile, el derecho de las obtenciones vegetales no se rige por la Ley № 19.039, de Propiedad Industrial, ni por la Ley № 17.336, de Propiedad Intelectual ${ }^{4}$, sino que se encuentra regulado por un régimen jurídico sui generis.

Este régimen jurídico sui generis se encuentra bajo el amparo del ADPIC ${ }^{5}$, en virtud del que los países miembros de la Organización Mundial de Comercio se hallan autorizados para "excluir de la patentabilidad las plantas y los animales excepto los microorganismos, y los procedimientos esencialmente biológicos para la producción de plantas o animales, que no sean procedimientos no biológicos o microbiológicos, sin embargo, los miembros otorgarán protección a todas las obtenciones vegetales mediante patentes, un sistema eficaz sui generis o por medio de una combinación de aquéllas y éste".

La protección de este derecho se confiere al obtentor de una nueva variedad vegetal ${ }^{6}$. En efecto, las variedades vegetales se definen por la ley como el "conjunto de plantas de un solo taxón botánico, o sea el elemento distintivo, del rango más bajo conocido que, con independencia de si responde o no plenamente a las condiciones para la concesión de un derecho del obtentor [que] puede definirse por la expresión de los caracteres resultantes de un cierto genotipo o de una cierta combinación de genotipos [y] distinguirse de cualquier otro conjunto de plantas por la expresión de uno de dichos caracteres por lo menos $^{\prime \prime}$. Y en escala las obtenciones vegetales se pueden definir como las va-

${ }^{3}$ El primer convenio UPOV data de 1961 y se firmó en París el 2 de diciembre. "Este convenio crea "La Unión para la Protección de las Obtenciones Vegetales (UPOV), como una Unión particular que se viene a sumar a la Unión General para la protección de la propiedad industrial creada por el Convenio de la Unión de París de 1883, y obliga a los Estados signatarios a proteger las variedades vegetales, siempre que éstas cumplan los requisitos de novedad, distinción, homogeneidad y estabilidad, y se le otorgue una denominación. La tutela puede tener lugar por medio de un título específico de protección, o por medio de un título de patente (adaptado a las peculiaridades recogidas en el convenio). Y en ambos casos esta protección se la denomina derecho de obtentor, terminología que en su momento fue criticada porque hacía alusión a su titular, más que al objeto de protección". GArCíA (2017a), pp. 66-67.

${ }^{4}$ Sobre los sistemas sui generis de protección, puede verse BiswajtT (2002), pp. 1-32; BLAKeney (2007), pp. 1-18.

${ }^{5}$ Artículo 27, numeral 3, letra b, del Acuerdo sobre los Aspectos de los Derechos de Propiedad Intelectual Relacionados con el Comercio, de la Organización Mundial de Comercio.

${ }^{6}$ El artículo $2^{\circ}$ reemplazar por: literal a) de la Ley $\mathrm{N}^{\circ} 19.342$, que regula derechos de obtentores de nuevas variedades vegetales, define al obtentor como "la persona natural o jurídica que, en forma natural o mediante trabajo genético, ha descubierto y, por lo tanto, logrado una nueva variedad vegetal".

${ }^{7}$ Artículo $2^{\circ}$, literal b, Ley № 19.342, que regula derechos de obtentores de nuevas variedades vegetales. 
riedades vegetales que cumplen con los requisitos necesarios para ser aptas para protección por medio del derecho del obtentor ${ }^{8}$, otorgándose una protección temporal, por un tiempo determinado ${ }^{9}$.

El derecho del obtentor se constituye de un modo originario mediante un acto de la autoridad administrativa. En efecto, el artículo $4^{\circ}$ de la Ley No 19.342 dispone que este derecho "se constituye por la inscripción en el Registro de Variedades Protegidas de un extracto del acuerdo del Comité Calificador que ordenó la inscripción y el otorgamiento del título correspondiente, el que debe contener una descripción objetiva de la variedad con referencia a los archivos técnicos".

La autoridad administrativa que otorga este derecho en Chile es el Servicio Agrícola y Ganadero, luego de un procedimiento en que se verifica que la variedad vegetal que se somete a examen es, efectivamente, nueva, distinta, homogénea y estable.

Dispone el artículo $9^{\circ}$ de la Ley $N^{\circ} 19.342$ que la variedad se considera nueva en el caso de que no haya sido objeto de comercio en el país y aquéllas que lo han sido sin el consentimiento del obtentor. Asimismo, se considerará nueva la variedad que ha sido objeto de comercio en el país con consentimiento del obtentor, pero por no más de un año. Del mismo modo se considerará nueva aquella que se ha comercializado en el extranjero con el consentimiento del obtentor, pero por no más de seis años, tratándose de árboles forestales, árboles frutales y árboles ornamentales y vides, y de cuatro años para las demás especies $^{10}$.

8 SÁncheZ (2008), p. 46. Sobre el concepto de obtención vegetal, puede verse Amat (2007), pp. 177 233; DutFieLd (2008), pp. 27-47.

9 De acuerdo con el artículo 11 de la Ley № 19.342, el plazo de protección, contado desde la fecha de inscripción del derecho del obtentor, será de 18 años para árboles y vides, y de 15 años para las demás especies.

${ }^{10}$ Con respecto a la novedad, MARCO (2017), pp. 290-291, indica que "la novedad como exigencia imperativa de protección de las variedades vegetales aparece expresamente contemplada en las diferentes normas que les resultan aplicables, las cuales se revelan de muy diversa índole como pues igualmente difieren en su configuración jurídica y ámbito geográfico, pese a que deban presentar la necesaria coordinación en cuanto a sus contenidos. De este modo, puede hablarse de la legislación en [el] ordenamiento interno, de disposiciones adoptadas en el marco del derecho de la UE y de importantes convenios internacionales en esta materia, y en unas y otros se incluyen distintos mandatos en relación con el requisito de la novedad. De ahí que pueda decirse que se reproduce en la regulación de las variedades vegetales la misma situación de círculos concéntricos (de menor a mayor extensión, nacional, supranacional internacional, respectivamente) que se observa en el régimen de muchos de los restantes títulos de propiedad industrial". Ahora bien, este autor sostiene que la entidad y alcance del requisito de la novedad en las obtenciones vegetales responde a otras finalidades y reviste particularidades con respecto a la novedad en las otras figuras de propiedad industrial. 
De acuerdo con el artículo 10 de la misma ley, la variedad es distinta "si puede distinguirse por uno o varios caracteres importantes de cualquiera otra variedad cuya existencia, al momento en que se solicite la protección, sea notoriamente conocida"11; es homogénea "si es suficientemente uniforme en sus caracteres pertinentes, a reserva de la variación previsible, considerando las particularidades de su reproducción sexuada o de su multiplicación vegetativa"12. Y es estable si sus caracteres esenciales se mantienen inalterados después de reproducciones o multiplicaciones sucesivas, o cuando el obtentor haya definido un ciclo particular de reproducciones o multiplicaciones al final de cada ciclo ${ }^{13}$.

\begin{abstract}
11 En cuanto a la relación de este requisito con la actividad inventiva en materia de patentes, se sostiene que "En ocasiones se ha intentado asimilar el requisito de la distinción al de la actividad inventiva en materia de patentes, argumentando que con la distinción se trata de proteger tan sólo las variedades que se aparten claramente de las ya conocidas. No obstante, y a mi juicio, semejante equiparación no es adecuada, porque la protección varietal por el sistema de la UPOV se aparta y es distinta del sistema de patentes, sin que sea necesario realizar equiparaciones ni buscar similitudes. y esto se ve contrarrestado por el hecho de que en la legislación española obtenciones vegetales remita de modo subsidiario la legislación de patentes, pues la regulación de los requisitos de concesión de un derecho de obtentor se realiza exhaustivamente por la normativa de obtenciones, sin que sea preciso acudir de modo subsidiario a la legislación de patentes. Por lo demás, mientras en materia de patentes la actividad inventiva implica que la invención no ha de resultar de manera obvia del estado de la técnica para un experto en la materia, el requisito de la distinción no atiende a la mayor o menor obviedad, ni a ningún tipo de actividad inventiva. Por el contrario, se trata de comprobar que la variedad se diferencia de las ya conocidas. De hecho la ausencia del requisito de altura inventiva en materia de obtenciones se constata si se tiene en cuenta que es posible proteger mediante un título de obtención vegetal una variedad aparecida de forma espontánea en la naturaleza (que posteriormente es desarrollada o puesta a punto), por lo tanto sin que medie altura o esfuerzo inventivo alguno". GarCía (2017b), pp. 357-358. Esta conclusión se refuerza en el derecho chileno, toda vez que la Ley $\mathrm{N}^{\circ} 19.342$ que regula derechos de obtentores de nuevas variedades vegetales, no se remite la Ley de Propiedad Industrial.
\end{abstract}

${ }^{12}$ Con respecto a este requisito se indica que "implica que los distintos ejemplos de la variedad presentan unas mismas características. En realidad, podría decirse que la homogeneidad o uniformidad supone que los individuos de una variedad deben ser indistinguibles entre sí. Y ello porque, si esto no fuera así, no sería factible determinar si un concreto individuo pertenece o no a la variedad, dificultándose la delimitación de la variedad con otras. Así las cosas, en realidad la homogeneidad opera como una especie de prerrequisito de la distinción, porque difícilmente podría hacerse una comparación de la variedad candidata con otras variedades si aquella no fuera homogénea". GARCíA (2017b), p. 385.

${ }^{13}$ Con respecto al requisito de la estabilidad se señala que "El requisito de la estabilidad implica la comparación, no de los distintos ejemplares de una misma variedad entre sí, sino la comparación de esos ejemplares con sus descendientes. Por eso se ha dicho con razón que la estabilidad supone la proyección en el tiempo de los caracteres que ya han sido objeto de valoración en el examen de la homogeneidad. De hecho, por regla general la variedad homogénea también es estable, como lo indica la propia UPOV [...]. El requisito de la estabilidad asimilado al de la susceptibilidad de aplicación industrial de las invenciones en materia de patentes. Dejando a un lado que, como ya he dicho, no considero procedente realizar semejantes comparaciones, no puede negarse que la estabilidad y la homogeneidad son requisitos que hacen que las variedades protegidas sean especialmente aptas para la agricultura a escala industrial. Y esto ha generado numerosas voces críticas que consideran que el sistema de protección de la UPOV no protege adecuadamente las variedades naturales o landraces, 
Ahora bien, la ley no define el derecho del obtentor, sino que más bien indica las facultades que confiere, las que consisten en someter a su autorización exclusiva: (1) la producción del material de multiplicación de la variedad; (2) la venta, la oferta o exposición a la venta de ese material; (3) la comercialización, la importación o exportación del mismo material; (4) el empleo repetido de la nueva variedad para la producción comercial de otra variedad, y (5) la utilización de las plantas ornamentales o de partes de dichas plantas que, normalmente, son comercializadas para fines distintos al de propagación, con vista a la producción de plantas ornamentales o de flores cortadas ${ }^{14}$.

Se puede apreciar que la ley evita dar directamente un concepto del derecho del obtentor y, en cambio, de un modo analítico establece las facultades que confiere utilizando conceptos técnicos.

Se establecen, asimismo, limitaciones al derecho del obtentor, que son básicamente dos, y que se encuentran también definidas técnicamente. La primera -conocida como el privilegio del agricultor y contemplada en el inciso final del artículo $3^{\circ}$ - dispone que no se entenderá vulnerado el derecho del obtentor por la utilización que haga el agricultor, en su propia explotación, de la cosecha del material de reproducción debidamente adquirido; sin embargo, este material no podrá ser publicitado ni transferido a cualquier título como semilla ${ }^{15}$. La segunda limitación -contemplada en el artículo $5^{\circ}$ y denominada excepción del obtentor-tiende a incentivar la creación de nuevas variedades, siempre que no sean esencialmente derivadas. Así, la ley dispone que "el derecho del obtentor sobre una variedad no impide que otra persona pueda emplearla para crear una nueva variedad, sin contar con la autorización del obtentor de la variedad primitiva que sirvió de medio para obtenerla. La nueva variedad así obtenida, si cumple con los requisitos legales, será reconocida a nombre de su obtentor ${ }^{16}$.

Se puede apreciar que esta reglamentación es eminentemente de carácter técnico y se encuentra conceptualmente relacionada con los derechos de propiedad industrial, a pesar de que, de acuerdo con el artículo 37 literal b) de la Ley $N^{\circ} 19.039$, de Propiedad Industrial, las obtenciones vegetales se encuentran excluidas de la patentabilidad, exclusión que se fundamenta en las diferencias que existen entre las obtenciones vegetales y las patentes. Aquí las diferencias

más propias de las prácticas agrícolas tradicionales y que no son tan estables, a la par que se fomenta una agricultura homogénea, con perjuicio para la diversidad biológica". GARCía (2017b), pp. 392-393.

${ }_{14}$ Artículo $3^{\circ}$, Ley $\mathrm{N}^{\circ}$ 19.342, que regula derechos de obtentores de nuevas variedades vegetales.

15 Sobre el privilegio del agricultor, puede verse Heimes (2010), pp. 1-55.

${ }^{16}$ Sin embargo, cuando la variedad original deba ser utilizada permanentemente para la producción de la nueva, se necesitará la autorización del obtentor de ella (artículo $5^{\circ}$, Ley № 19.342). Sobre la excepción del obtentor, puede verse JANIS (2002), pp. 5-13. 
entre ambos institutos son de diversa índole y, siguiendo a Gómez Segade ${ }^{17}$, estas diferencias se estructuran por la posición frente a la biodiversidad ${ }^{18}$, por el objeto de protección ${ }^{19}$, por los requisitos sustantivos de protección ${ }^{20}$, por el requisito formal de la denominación ${ }^{21}$, por las limitaciones del derecho del titular de la obtención vegetal y por la duración de este derecho.

\section{Fundamento}

Históricamente, la innovación en el campo del fitomejoramiento se incrementó a partir del siglo XVIII, cuando se logra conocer la diferencia entre la reproducción asexual y la reproducción sexual de los vegetales. Con la

17 Gómez (2017), pp. 120-129.

18 "Con carácter general la gran diferencia entre el sistema de patentes y el de obtenciones vegetales radica en la filosofía de fondo que se oculta bajo los dos sistemas y de la que derivan en buena medida muchas de las ulteriores diferencias concretas. En efecto, la patente desde el primer momento nace dominada por una lógica industrial y por tanto intenta fortalecer lo más posible el derecho de exclusiva y la posición del titular del derecho; por consiguiente, muchos desarrollos de la regla técnica se consideran dependientes de la invención amparada por la patente, y su empleo, aunque en último término tuviera como meta conseguir una nueva invención patentable, constituiría una infracción de la patente. La obtención vegetal, por el contrario, ha sido concebida para aplicarse a materia viva desde el primer momento. Por eso en la moderna legislación sobre obtenciones vegetales se considera esencial garantizar que continúe la mejora genética de las especies vegetales, e impedir un efecto bloqueo como el que surge por efecto de la patente. Se favorece el acceso a los recursos genéticos mediante la denominada excepción del obtentor que permite a un obtentor acceder libremente a cualquier variedad previa, aunque esté protegida, para conseguir a partir de la misma una nueva variedad vegetal". Gómez (2017), p. 120.

${ }^{19}$ Se señala que "el objeto de protección de las patentes es amplísimo pues puede consistir en cualquier invención en cualquier sector de la técnica. En cambio, el objeto de protección de las obtenciones vegetales es mucho más limitado y concreto porque podría estar constituido tan sólo por variedades vegetales [...]. El limitado objeto de protección de las obtenciones vegetales viene condicionado por la naturaleza del derecho que protege las obtenciones vegetales, que es un derecho ad hoc o sui generis frente al carácter general del derecho de patentes". Gómez (2017), p. 121.

${ }^{20}$ Se sostiene que "son enormes las diferencias entre los requisitos materiales para obtener una patente o un certificado de obtención vegetal. Como es sabido, para que pueda obtenerse una patente deben concurrir los tres requisitos de patentabilidad de novedad, actividad inventiva y susceptibilidad de aplicación industrial. Pues bien, para obtener una obtención vegetal no se precisan ni susceptibilidad de aplicación industrial ni actividad inventiva; en consecuencia puede obtenerse un certificado de obtención vegetal para una variedad natural que el solicitante simplemente ha descubierto por casualidad, en cuyo caso desde luego el derecho de exclusiva no se otorga para premiar una creación que no existe sino para estimular la investigación agraria y el fitomejoramiento". Gómez (2017), p. 122.

${ }^{21}$ Maroño (2017), p. 396, destaca que "el reconocimiento de un derecho de obtención vegetal va indisolublemente ligado a la atribución de una determinada denominación a la nueva variedad protegida, denominación que deberá ser propuesta por el obtentor. De hecho, las regulaciones sobre títulos de obtención vegetal de nuestro entorno exige la atribución de una denominación para cada nueva variedad ajustada a lo requerido por la normativa vigente. Y la importancia que se le da a este requisito es tal que, sin la asignación de una denominación a la variedad, no podrá concederse el título de obtención vegetal". 
reproducción sexual surge la alternativa de cruzar las plantas de una manera artificial y controlada, para enseguida seleccionar los individuos más ventajosos de acuerdo con las características que se estimen valiosas, y luego intentar que se mantengan tales características estables en sucesivas generaciones. Es decir, se trata de generar variaciones genéticas en las plantas para obtener resultados queridos y a continuación seleccionar los individuos para su reproducción ${ }^{22}$.

Ahora bien, la generación de semillas y de los demás materiales de reproducción de variedades vegetales es una actividad que requiere de altos grados de biotecnología ${ }^{23}$, de modo que el desarrollo de una nueva variedad vegetal necesita de grandes inversiones, en tiempo y dinero ${ }^{24}$. En este sentido, Gómez Segade destaca que el desarrollo y obtención de nuevas variedades vegetales implica una considerable inversión en tiempo y recursos financieros que en términos generales pocas veces será inferior a los dos millones de euros por variedad, y, así, no resulta posible amortizar la gran inversión de recursos requeridos con la sola comercialización y venta del material obtenido, a causa de que la naturaleza del vegetal permite una multiplicación en general ilimitada y constantemente susceptible de comercializarse ${ }^{25}$, lo que implica que terceros que no han realizado la correspondiente inversión pueden fácilmente utilizar la variedad vegetal para su reproducción y comercialización.

Como señala García Vidal, en los casos de variedades susceptibles de reproducción asexual o vegetativa bastará con hacerse con un esqueje de la variedad para poder obtener individuos clónicos y, en consecuencia, exactamente iguales ${ }^{26}$. En este sentido, se ha dicho que el que se hace con material de reproducción de una variedad se queda también con la "fábrica" de sucesivos individuos de la misma planta, recalcándose de este modo la necesidad de mayor de protección que se requiere en este campo en relación con otros sectores productivos, toda vez que, por ejemplo, un kilogramo de algún producto químico será invariablemente un kilogramo; en cambio, un kilogramo de papas es potencialmente una ilimitada cantidad de plantas de la misma variedad ${ }^{27}$.

22 Gómez (2017), p. 113.

${ }^{23}$ En este sentido, Rapela (2006), p. 135.

${ }^{24}$ Toda vez que el proceso de obtención de una nueva variedad vegetal oscila alrededor de los diez años y, como se destaca, la inversión global en el sector también es muy notable. Así, por ejemplo, el presupuesto de la investigación privada en innovación vegetal en los EE.UU. en el año 2011 fue de 4.500 millones de dólares. Le BuANEC (2011), p. 12.

25 Gómez (2017), pp. 112 y 113.

${ }^{26}$ García (2017a), p. 51.

27 Wuesthoff (1957), p. 49. 
Se argumenta que de este modo surge la necesidad de protección de la labor que implica el fitomejoramiento mediante obtenciones vegetales, ya que la alta inversión requerida no puede amortizarse simplemente con la mera venta del material obtenido, porque la naturaleza permite una multiplicación constante e ilimitada de la variedad ${ }^{28}$.

De esta manera, la creación por ley del derecho del obtentor vegetal tiene por objetivo retribuir, por un tiempo determinado, el trabajo de investigación y desarrollo y los recursos que se requieren para obtener una variedad vegetal que cumpla con los requisitos de protección. ${ }^{29}$.

Así, se ha dicho que la concesión de un derecho de exclusiva al titular de la variedad no solo tiene por objeto premiar su innovación, sino también estimular la obtención y divulgación de nuevas variedades vegetales, y que por esto se crea un sistema de protección que trata de mantener el equilibrio entre el interés general en que se desarrollen nuevas variedades y el interés del obtentor, y para conseguir estos objetivos, el mecanismo más eficaz sería la concesión de un derecho de propiedad industria ${ }^{30}$.

\section{Objeto de protección}

Al tratarse de un derecho de propiedad industrial o intelectual (en sentido amplio) se ha asimilado, como se verá, la protección del derecho del obtentor vegetal al derecho de propiedad. Ahora bien, se indica que el concepto de derecho de propiedad con respecto a la propiedad industrial e intelectual se concibe en una acepción de mayor amplitud que la usual, prácticamente asimilable a la categoría de un derecho de titularidad sobre un derecho subjetivo absoluto, cuyo objetivo sería dejar clara la afirmación de que se trata de un derecho individual en lugar de un privilegio concedido por autoridad, y sin mayores implicancias en las características del objeto de este derecho ${ }^{31}, \mathrm{y}$ "ello porque son evidentes las diferencias entre la protección otorgada por la propiedad industrial e intelectual y el derecho de propiedad que recae sobre cosas corporales" ${ }^{\prime 2}$. De este modo, "la

${ }^{28}$ GÓmez (2017), pp. 112 y 113.

29 Sobre los cuestionamientos generales a esta institución y sus relaciones con los recursos genéticos y conocimientos tradicionales, puede verse KESAN (2001), pp. 1-42.

30 Gómez (2017), p. 113: "El monopolio temporal que implica el derecho de propiedad industrial estimula la competencia porque permite al obtentor recuperar las inversiones realizadas y evita que un tercero se apropie de la creación sin gasto ni compensación alguna. El equilibrio entre los intereses del creador y los de la generalidad se manifiesta no sólo en la temporalidad de la exclusiva sino también en la necesaria concurrencia de ciertos requisitos para que nazca el derecho de exclusiva".

31 En este sentido, Curto (2017), pp. 788-789, Gómez (1974), p. 71.

${ }^{32}$ Curto (2017), p. 789. 
principal diferencia radicaría en que las cosas corporales preexisten al derecho de propiedad, mientras que en el caso de la propiedad industrial e intelectual es el propio Derecho el que establece los requisitos del objeto sobre el que recaen" ${ }^{\prime \prime 3}$.

Fuera de toda duda, se puede decir que el derecho del obtentor es un derecho real que consiste un derecho exclusivo de explotación de la variedad vegetal. Esta noción del derecho del obtentor como derecho real, y dada la carencia de normas que regulen completamente su régimen jurídico en la ley especial que rige la materia en Chile, posibilita que, para la determinación de las reglas en los aspectos donde hay vacíos, se pueda recurrir, al menos como una alternativa a priori, a las normas generales dispuestas en el ordenamiento jurídico en relación con los derechos reales, o bien a las normas especiales de ordenamientos análogos de propiedad intelectual ${ }^{34}$ e industrial. Ahora bien, el artículo 37 de la Ley $\mathrm{N}^{\circ} 19.039$, de Propiedad Industrial, establece que las variedades vegetales no se consideran invención y quedan excluidas de la protección por patente, no existiendo norma que ordene la aplicación de esta ley en materia de obtenciones vegetales.

Específicamente, el objeto de protección consiste precisamente en una obtención vegetal y, desde el punto de vista intelectual, se protege la información genética contenida en la nueva variedad, contando el titular del derecho con las facultades contempladas en el artículo $3^{\circ}$ de la Ley $N^{\circ} 19.342$, ya descritas más arriba, que consisten sintéticamente en conferir derechos de explotación de esa información genética, de un modo exclusivo y excluyente, para la reproducción y comercialización de la variedad vegetal protegida, por un tiempo determinado, y salvas las excepciones que establece la ley.

Las excepciones específicas, y que son figuras singulares en este derecho, son típicamente dos: el denominado privilegio del agricultor ${ }^{35}$ y la llamada excepción del obtentor ${ }^{36}$.

\footnotetext{
33 VICENT (1991), p. 13.

${ }^{34}$ La Corte de Apelaciones de San Miguel, № 1418-2012, de 14 de mayo de 2014, ha aplicado supletoriamente la Ley № 17.336, sobre Propiedad Intelectual.

35 Se explica que "por virtud de esta excepción, el agricultor puede, sin necesidad de la previa autorización del obtentor, emplear en su propia explotación y con fines de propagación, el producto de la cosecha que haya obtenido de la plantación de material de propagación de una variedad que esté protegida por un derecho de obtenciones vegetales [...]. Se acepta pacíficamente que ya desde épocas anteriores a la puesta en marcha de estos sistemas era práctica habitual que los agricultores reservasen material de reproducción de los productos obtenidos de sus cosechas -particularmente cuando éstos eran de buena calidad- con el fin de realizar su siembra en el siguiente ciclo de cultivo; práctica que se mantuvo después del reconocimiento de derechos de propiedad industrial a los obtentores de las variedades vegetales a las que correspondía el material de propagación empleado por aquellos". BOtana (2017), pp. 611-612.

${ }^{36}$ García (2017c), p. 639, indica que "de conformidad con este principio, cualquier persona puede usar una variedad vegetal protegida para obtener una segunda variedad vegetal que derive de ella.
} 
Ahora bien, sobre el contenido del derecho del obtentor con respecto a plantas y semillas en cuanto bien material, es decir, con respecto a las cosas corporales en que se manifiesta la variedad vegetal, Sánchez Gil ha dicho que tiene por objeto o renace con cada reproducción de la obtención vegetal protegida, lo que implica que por cada nuevo ciclo de cultivo de la variedad vegetal se devenga una nueva remuneración o canon, más no implica que el obtentor se haga dueño de las plantas o de lo cosechado ${ }^{37}$.

Ahora bien, la materialidad o inmaterialidad del bien que es finalmente objeto de protección, es asunto de controversia conceptual doctrinaria comparada, que se manifiesta también implícitamente en la jurisprudencia chilena. Esta cuestión influye directamente en los remedios para proteger tal derecho, problema que entre nosotros se agrava atendido que no existen mecanismos especiales de acciones civiles como en otras leyes nacionales de propiedad industrial o intelectual.

Según Gómez Segade, "quien consigue una nueva obtención vegetal crea un bien inmaterial producto de la mente humana, que no debe confundirse con la planta o variedad vegetal en que se plasma exteriormente esa creación" ${ }^{\prime 38}$. Prosigue señalando que el bien inmaterial protegido por el derecho sobre obtenciones vegetales es un derecho de propiedad industrial con características semejantes a las de otros derechos de propiedad industrial y, entre estas características, cabe destacar el carácter registral, la exclusividad, la temporalidad y la territorialidad ${ }^{39}$.

En otro sentido, se ha dicho que el objeto de protección tiene un carácter tangible, lo que determina la naturaleza jurídica de la protección que se otorga. Al respecto, Curto indica que de la "definición de la misma en los textos legales se deduce claramente que el objeto de protección en este supuesto, a diferencia de lo que ocurre en relación con otras instituciones incardinadas en la propiedad industrial y la propiedad intelectual, como las marcas, las patentes o el derecho de autor, no es un bien inmaterial, sino un bien material. En efecto, el derecho del obtentor recae sobre un determinado material vegetal de rango

Además, el segundo obtentor puede comercializar las nuevas variedades a las que ha llegado a partir de la variedad inicial, sin autorización y sin pago de remuneración alguna al titular de la variedad".

37 SÁnCHeZ (2008), p. 135.

38 Gómez (2017), pp. 111 y 112.

39 Gómez (2017), pp. 111 y 112. En el mismo sentido, Maroño (2017), p. 395, indica que "El derecho de obtentor es un derecho de propiedad industrial que tiene por objeto proteger la innovación que supone la aportación, al universo de las variedades vegetales, de una variedad que sea nueva, estable, distinta y homogénea. De ahí que el derecho de propiedad industrial sobre una obtención vegetal se dirija esencialmente a evitar utilizaciones no autorizadas del material protegido, que vulnere el derecho de uso en exclusiva que se reconoce al titular de la obtención vegetal". 
taxonómico inferior, del que el solicitante del título debe estar en posesión, a fin de poder cumplir con los requisitos formales establecidos para la concesión de la protección jurídica". Y agrega que "pese a la materialidad del objeto de protección, en la medida en que es el resultado de la innovación desarrollada por el obtentor, o del descubrimiento y desarrollo de un determinado material vegetal, el legislador, nacional e internacional, ha optado por conferirle una protección que puede ser encuadrada dentro de la propiedad industrial"40. Sin perjuicio de lo dicho, en esta doctrina igualmente se sostiene que el derecho del obtentor se encuadra dentro de la llamada propiedad industrial y que, en consecuencia participa, de las características de esta ${ }^{41}$, por lo que existe consenso en que se trata de un derecho de propiedad industrial (o intelectual en sentido lato), con el matiz de que esta última doctrina entiende que el objeto de la protección también recae en un bien material o corporal, o al menos se extiende a este. En el marco de esta discusión, los escasos fallos de los tribunales chilenos se han inclinado por uno u otro sentido.

La Corte de Apelaciones de San Miguel ${ }^{42}$, con base en el inciso II, del artículo $3^{\circ}$ de la Ley $N^{\circ} 19.342$, que dispone que "el derecho del obtentor se puede ejercer sobre todos los géneros y especies botánicos y se aplica, en general, sobre la planta completa, comprendiendo todo tipo de flores, frutos o semillas y cualquier parte de la misma que pueda ser utilizada como material de multiplicación", ha fallado que las cosas corporales (en este caso se trataba de plantas) caben dentro del objeto de protección del derecho del obtentor vegetal ${ }^{43}$. Para llegar a tal conclusión, la citada corte sostiene que "el derecho del obtentor es una forma de propiedad intelectual que se reconoce a los creadores de nuevas variedades vegetales a objeto de permitirles la explotación exclusiva de su creación, por un tiempo determinado; poseyendo características comunes con las otras formas de propiedad intelectual. La propiedad intelectual se encuentra protegida tanto por la legislación interna, como por los diversos Tratados de Libre Comercio, como por la adhesión a múltiples acuerdos internacionales sobre el tema".

Esto es, califica a este derecho como un derecho de propiedad intelectual, pero no en el sentido lato del término, sino que para aplicar, ante la ausencia de acción civil, la Ley $N^{0}$ 17.336, de Propiedad Intelectual, que regula el derecho de autor y los derechos conexos, basándose en que esta ley protege también las

\footnotetext{
${ }^{40}$ Curto (2017), p. 786.

41 Curto (2017), p. 788.

42 Corte de Apelaciones de San Miguel, No 1418-2012, de 14 de mayo de 2014.

43 Corte de Apelaciones de San Miguel, No 1418-2012, de 14 de mayo de 2014.
} 
creaciones en el ámbito científico, en las que -parece ser el entendimiento de la corte- se subsumen las obtenciones vegetales, soslayando, entre otros aspectos, que es la misma ley la que dispone que los derechos que rige se adquieren por el "solo hecho de la creación de la obra", cuestión que, como se ha visto, está lejos de estar presente en materia del derecho del obtentor. Y es más, la corte lleva al extremo este entendimiento, al fallar que "en consideración a la clase de propiedad de que se trata, especie de propiedad intelectual, la protección del derecho se le concede [al obtentor] para la explotación exclusiva de su creación y derechos conexos, pudiendo reivindicar tanto el material genético, como la reproducción de la especie obtenida, como sus plantas, frutos y flores, cuando se hubieren obtenido sin su autorización expresa, como ha ocurrido en la especie. El reducir la protección del derecho solo a la creación y no a la reproducción de esta haría ineficaz el resguardo, porque permitiría a quienes utilizaren las especies reproducidas de ese material genético, a obtener los beneficios que corresponden al creador. De esa forma, el pretender que solo corresponderían al obtentor la indemnización de los perjuicios que el uso de su obra le ocasionare, le resta la calidad de dueño absoluto de esta y permitiría su aprovechamiento por terceros no autorizados".

Huelga señalar el palmario error conceptual en que incurrió este fallo, al referirse a derechos conexos en materia de obtenciones vegetales, cuestión improcedente. Sí cabe exponer la consecuencia de todo este razonamiento errado: se confirió acción reivindicatoria sobre bienes corporales consistentes en plantas y frutos cosechados de la variedad de que se trataba; en este caso, de la variedad de ciruela Constanza ${ }^{44}$.

Cabe destacar que el voto disidente de este fallo estuvo por rechazar la acción reivindicatoria, sobre la base de que del "análisis de las disposiciones establecidas en la Ley № 19.342 que regula los derechos sobre variedades vegetales en especial aquellos que regulan los derechos del obtentor-artículos $1^{\circ}$, $2^{\circ}, 3^{\circ}, 4^{\circ}$ y $5^{\circ}$ - no puede desprenderse que su titular sea dueño de los arboles cuyo patrón proviene de la variedad vegetal protegida a favor del actor, sino que para ello deben aplicarse las reglas generales sobre la accesión de cosa mueble a inmueble, y el derecho que le asiste en este caso al obtentor es de accionar criminalmente de conformidad a los tipos penales que dicha ley contempla y sin perjuicio del derecho de ser indemnizado por el infractor, de acuerdo a las reglas generales" ${ }^{\prime 45}$. La disidencia, en suma, señala que no procede la acción

${ }^{44}$ La Corte Suprema, si bien dejó firme este fallo, no se pronunció sobre el fondo del asunto. Corte Suprema, № 25682-2014, de 10 de agosto de 2015.

${ }^{45}$ Voto disidente del abogado integrante José Miguel Puelma Barriga en fallo de la Corte de Apelaciones de San Miguel, N 1418-2012, de 14 de mayo de 2014. La disidencia agrega que "la debida aplicación 
reivindicatoria en la materia, sobre la base de distinguir la obtención vegetal de las cosas corporales que portan la genética de la variedad vegetal.

El Tribunal de Propiedad Industrial ${ }^{46}$, conociendo de una acción de nulidad de un registro de una obtención vegetal, finalmente la acogió, en un fallo en que se aprecia claramente la distinción entre la protección a la propiedad intelectual (en sentido lato) y los cosas corporales, esto es, por lo general, plantas o semillas, en que se concreta la información genética que se protege mediante el derecho del obtentor, el que no entiende como una especie de propiedad, sino como un derecho que llama de protección.

Ahora bien, sea cual fuere el alcance del derecho del obtentor en cuanto a los bienes corporales en que se incardina, la ley presenta un notable y sensible vacío: no contempla una acción civil directa para detener el uso no autorizado del material de multiplicación consistente en la obtención vegetal, ni tampoco regula lo referente a la indemnización de perjuicios. Ambos tópicos se regulan expresamente en otros ordenamientos que siguen el convenio UPOV ${ }^{47}, y$, si bien con matices, tienden al objetivo del cese de la explotación no autorizada de la nueva variedad vegetal protegida a favor de su obtentor. Lo mismo que las leyes patrias que regulan el derecho de autor y los derechos de propiedad industrial, que confieren acción civil especial para cubrir ambas pretensiones.

\section{Conclusión}

La carencia de un régimen jurídico exhaustivo del derecho sobre las obtenciones vegetales, en especial la importante falta de la reglamentación de las acciones civiles protectoras de este derecho, ha comportado que los tribunales de justicia no tengan claridad respecto de la determinación del objeto de protección del derecho del obtentor vegetal. Todo esto debido a que, como se dejó en evidencia en el desarrollo de este trabajo, el ámbito de las obtenciones

de las normas de los artículos 889 y 898 ambos del Código Civil, nos llevan a concluir que el actor no era dueño de lo que pretende reivindicar -árboles frutales y sus frutos-, razón suficiente para rechazar el libelo pretensor. Pero además de acuerdo a la prueba rendida tampoco se encuentra cumplido el requisito de la acción se haya dirigido en contra del que posee actualmente la cosa, que en este caso eran los propietarios del inmueble a la época de haberse entablado la demanda, lo que fue acreditado por medio de inscripciones dominicales, que eran sociedades distintas a las demandadas. Con respecto a la aplicación en este caso de la acción contemplada en el artículo 898 del Código Civil, ésta no procede por cuanto -como se ha explicado- al no ser el actor el propietario de los árboles frutales objeto de la reivindicación, no es posible otorgarle el derecho que esa disposición".

46 Tribunal de Propiedad Industrial, № 1615-2012, de 28 de agosto de 2014.

47 Por ejemplo, en España Ley № 3/2000, de 7 de enero de 2000, de Régimen Jurídico de la Protección de las Obtenciones Vegetales, y en México, aunque de manera inorgánica, en la Ley Federal de Variedades de 25 de octubre de 1996. 
vegetales es complejo, de alto contenido biotecnológico, dotado por lo mismo de una reglamentación eminentemente técnica, pero incompleta, respecto de la que cabe, en principio, aplicar los remedios del derecho privado común, aunque estos resultan insuficientes para dar respuesta a los problemas que presenta la materia.

\section{Bibliografía CitADA}

Amat Llombart, Pablo (2007): "Concepto, Contenido y Límites del Derecho de Obtentor de Variedades Vegetales según la Ley N 3/2000 de 7 de enero y Real Decreto No 1.261/2005 de 21 de octubre", en: La propiedad industrial sobre obtenciones vegetales y organismos transgénicos (Madrid, Tirant lo Blanch), pp. 177-233.

Barrera Miranda, Alejandra, y Donoso Cáceres, Alejandra (2013): "La aprobación del UPOV 91: La biodiversidad como objeto de propiedad intelectual. Análisis crítico en justicia ambiental", en: Revista de Derecho Ambiental (No 5), pp. 219-234.

BISWAIT, Dhar (2002): "Sistemas sui generis para la protección de variedades vegetales. Opciones bajo el Acuerdo sobre los ADPIC". Disponible en: https://quno.org/sites/default/files/resources/Sui-Generis-Systems-for-PlantVariety-Protection-Spanish.pdf [visitado el 4.07.2017].

Blakeney, Michael (2007): "Plant Variety Protection, International Agricultural Research, and Exchange of Germplasm: Legal Aspects of Sui Generis and Patent Regimes, Intellectual Property Management in Health and Agricultural: A Handbook of Best Practices". Disponible en: http://www.iphandbook.org/ handbook/ch04/p07/ [visitado el 5.09.2017].

Botana Agra, Manuel (2017): "La excepción en beneficio del agricultor", en: García Vidal, Ángel (director), Derecho de las obtenciones vegetales (Madrid, Tirant lo Blanch), pp. 611-635.

Curto Polo, M. Mercedes (2017): "Cotitularidad y transmisión del derecho del obtentor", en: García Vidal, Ángel (director), Derecho de las obtenciones vegetales (Madrid, Tirant lo Blanch), pp. 785-818.

Dutfield, Graham (2008): "Turning Plant Varieties into Intellectual Property: The UPOV Convention, The Future control of food, ed. Tansey y Rajotte, Erathscan/IDRC". Disponible en: http://www.idrc.ca/es/ev-119946-201-1DO_TOPIC.html [visitado el 2.09.2018].

García VIDAL, Ángel (2017a): “El sistema de protección de las variedades vegetales", en: García Vidal, Ángel (director), Derecho de las obtenciones vegetales (Madrid, Tirant lo Blanch), pp. 46-109. 
(2017b): "Los requisitos de la distinción, la homogeneidad y la estabilidad", en: García Vidal, Ángel (director), Derecho de las obtenciones vegetales (Madrid, Tirant lo Blanch), pp. 349-394.

(2017c): "El privilegio del obtentor y las variedades esencialmente derivadas", en: García Vidal, Ángel (director), Derecho de las obtenciones vegetales (Madrid, Tirant lo Blanch), pp. 638-643.

Gómez Segade, José Antonio (2017): "Diferencias entre el sistema de patentes y el sistema de protección de las obtenciones vegetales", en: García Vidal, Ángel (director), Derecho de las obtenciones vegetales (Madrid, Tirant lo Blanch), pp. 111-129.

Gómez Segade, José Antonio (1974): El secreto industrial (know-how). Concepto y protección (Madrid, Tecnos).

Heimes, Rita (2010): "Post-Sale Restrictions on Patented Seeds: Which Law Governs?, Wake Forest Intellectual Property Law Journal". Disponible en: http://papers.ssrn.com/sol3/papers.cfm?abstract_id=1593230 [visitado el 11.07.2017].

JANIS, Mark (2002): "Intellectual Property Issues in Plant Breeding and Plant Biotechnology, Biotechnology, Gene Flow, and Intellectual Property Rights: An Agricultural Summit, Purdue University". Disponible en: http://www. agriculture.purdue.edu/arp/ag_summit.pdf [visitado el 15.06.2017].

KESAN, Jay (2001): "Intellectual Property Protection and Agriculture Biotechnology a Multidisciplinary Perspective", en: American Behavioral Scientist (Vol. 44, $\left.N^{\circ} 3\right)$, pp. 464-503.

LE BUANEC, Bernard (2011): "La evolución del fitomejoramiento y de la protección de las obtenciones vegetales", en: AA. VV., Simposio sobre el fitomejoramiento para el futuro (UPOV, Ginebra), pp. 12-22.

Marco ArCalÁ, Luis (2017): "Variedades vegetales: El requisito de novedad", en: García Vidal, Ángel (director), Derecho de las obtenciones vegetales (Madrid, Tirant lo Blanch), pp. 290-348.

Maroño Gargallo, María del Mar (2017): "La denominación de las variedades protegidas con un título de obtención vegetal", en: García Vidal, Ángel (director), Derecho de las obtenciones vegetales (Madrid, Tirant lo Blanch), pp. 395-345.

Moronı BÁez, Paz (2014): "Marco jurídico chileno sobre protección de variedades vegetales", en: Actualidad Jurídica, Universidad del Desarrollo ( $\left.\mathrm{N}^{\circ} 30\right)$, pp. 327-346.

Rapela, Miguel Ángel (2006): "Excepción y derecho del agricultor. Origen y desarrollo", en: Rapela, Miguel Ángel (director), Innovación y propiedad 
intelectual en el mejoramiento vegetal y biotecnología agrícola (Buenos Aires, Heliasta-Universidad Austral), pp. 135-149.

Rojas NeIRA, Jorge (2013): "Acceso a recursos genéticos y conocimiento tradicional. Elementos críticos para una política y legislación en Chile", en: Revista de Derecho Ambiental (№ 4), pp. 117-140.

Sánchez GIL, Olga (2008): La protección de las obtenciones vegetales. El privilegio del agricultor (Madrid, Ministerio de Medio Ambiente, Medio Rural y Marino).

Schmitz Vaccaro, Christian (2005): Propiedad intelectual a la luz de los tratados de libre comercio (Santiago, Editorial LexisNexis Chile).

VICENT Chulí, Francisco (1991): Compendio crítico de derecho mercantil, 3 a edición (Barcelona, Bosch), tomo I, Vol. 1.

WuesthOfF, Freda (1957): Patentschutz für Pflanzen, en: Grur Journal ( $\left.^{\circ} 2\right)$, pp. 49-56.

\section{NORMAS JURÍDICAS CITADAS}

Código Civil chileno.

Ley $N^{\circ} 19.342$, que regula derechos de obtentores de nuevas variedades vegetales. Diario Oficial No 35.006, 3 de noviembre de 1994.

Ley N N 17.336, sobre Propiedad Intelectual. Diario Oficial, 2 de octubre de 1970. Ley № 19.039, sobre Propiedad Industrial. Diario Oficial, 25 de enero de 1991. Convenio de la Unión Internacional para la Protección de las Obtenciones Vegetales, de 1978.

Ley $N^{\circ} 3 / 2000$, de Régimen Jurídico de la Protección de las Obtenciones Vegetales, de 7 de enero de 2000 (España).

Ley Federal de Variedades, de 25 de octubre de 1996 (México).

\section{JURISPRUDENCIA CITADA}

Sociedad Agrícola Santa Beatriz y Sociedad Agrícola Santa Alicia Limitada con Godoy Puyol Agrónomos Asociados (2014): Corte Suprema 10 agosto 2015 (casación) sin publicar.

Godoy Puyol Agronomía Asociados y Otro con Fantuzzi Alliende Mario y Otros (2014): Corte de Apelaciones de San Miguel 14 mayo 2014 (casación) sin publicar.

Sociedad Agrícola Santa Beatriz y otro con Godoy Puyol Agrónomos Asociados (2014): Tribunal de Propiedad Industrial 28 agosto 2014. 
